\section{A Bibliography on Parallel Parsing}

Henk Alblas, Rieks op den Akker, Paul Oude Luttighuis, and Klaas Sikkel Department of Computer Science, University of Twente, P.O. Box 217, 7500 AE Enschede, The Netherlands \{alblas, infrieks, oudelutt, sikkel\}ecs.utwente.nl

\section{Abstract}

This bibliography on parallel parsing and recognition of context-free languages covers most of the important publications in this area of computer science and natural language processing. The list of publications is preluded by an introduction which presents an overview of the field.

\section{Introduction}

This bibliography on parallel parsing is a modified and compacted version of the annotated bibliography of Op den Akker et al.[1992], from which all annotations have been removed, as well as preand re-publications and papers that were not available to us. Readers interested in the complete annotated version are invited to contact the authors.

During the last few years we have seen a trenendous number of papers related to parsing and recognition of (mostly very restricted) context-free grammars in the area of connectionist or neura networks. These networks do not only parsing or recognition but simulate recognition of semantical and pragmatical aspects of words, or phrases in natural language utlerances as well. The results and pragmatical aspects of words, or phrained in this area are not covered.
obta

If we look at the publications in the area of parallel parsing we may distinguish four differen approaches or interests.

- There are papers which are rather theoretically concerned with language recognition and sometimes parsing. These papers introduce several models of parallel computation and try to relate these models with other models or with well-known classes of languages. One of the questions considered in these papers is for which subclasses of the class of context-free languages recognition can be optimally parallelized.

- Several papers concern parallelization of general context-free language parsing methods. Here we find methods for parallel CYK and Earley parsing or variants of these and proposals for systolic array or VLSI implementations of these parsing methods. Especially in the field of natural language processing emphasis is on these general parsing methods. Some papers leces allention to necthods for language specification suitable for parallel parsing.
- This bibliography covers also papers which consider parallel parsing in a broader context of complete parallel compiler systems. In the following we will give a short overview of these different fields of interest.

\section{Parallel Parsing of Programming Languages}

This section describes work done in the area of parallel lexing and parsing of programming languages. Papers in this area can be categorized on the basis of several criteria:

1. compilation phase: lexing, parsing or both;

2. language class: arithmetic expressions, bracket matching, more general language classes;

3. parallel machine model: vector computer, shared memory model (SIMD, P-RAM), hypercube, mesh-, shuffle-, cube-connected and cube-connected cycles;

4. starting-point: sequential algorithms, or not;

5. number of processors: fixed, or depending on grammar or string size;

6. decomposed domain: grammar or string decomposition.

We will not make a full 6-dimensional split-up of this area according to these six criteria. Instead our division of the subject obeys a tree-like structure, so that some of the sub-areas may be scattered over this tree. Our main split-up concerns the compilation phase under consideration.

\section{Lexing}

Early contributions, like Lincoln[1970] and Donegan and Katzke[1975] show techniques for lexical analysis on a vector computer. Another SIMD lexing algorithm appears in Asthagiri and Potter[1992]. In Hillis and Steele[1986], the parallel prefix sum algorithm (Ladner and Fischer[1980]) is applied to finite automata to yield an optimally parallel regular recognizer. Implementations of this lexing technique can be found in Steele and Hillis[1986] and Hill[1992]. Lewke[1990] uses dedicated hardware for parallel lexing. Different approaches to parallel lexical analysis appear in McDonald and Wendelborn[1990].

\section{Lexing and parsing}

Pronina and Chudin[1975] use a pipeline between a lexing and a parsing processor.

\section{Parsing}

In parallel parsing, two approaches may be distinguished. The first approach uses a decomposition of the grammar and lets processors be responsible for parts of the grammar, that is, for parsing as far as productions in its own part of the grammar are concerned. The other approach just distributes the string over prexessors, which all parse their part according to the whole grammar. An advantage of the first method is, that actual parsing is facilitaled, because the string can be cut at convenient 
places. Advantages of the second method are that distribution of the string is easy and that the distribution can freely be done in such a way that processors get more or less equally long parts of the string to handle.

\section{Grammar decomposition.}

Many papers using the grammar-decomposition approach originate from France. In Baccelli and Fleury[1982], Fleury[1983] and Baccelli and Mussy[1986], the string is partitioned according to some decomposition of the grammar. Partitioning is performed (sequentially) by a host process. After that different processes perform local parsing (Baccelli and Fieury [1982]) or interpreting (Baccelli and Mussy[1986]). A similar idea appears in Khanna and Ghafoor[1990].

\section{String decomposition.}

The string decomposition approach is used by the vast majority of papers on this topic. Probably, this is because it allows for using arbitrary numbers of processors, that is, a number not fixed (by the algorithm or the grammar). Again, we split up these papers in several groups.

1. papers that use bracket matching as (part of) their algorithm;

2. papers that discuss parallel bottom-up parsing;

3. papers that use the parallel prefix sum algorithm;

4. other papers

Bracket matching. In the early 1980 's, work was done on parallel parsing of arithmetic expresBracket matching. In the early 1980 's, work was done on parallel parsing of arither
sions. In Dekel and Sahni[1983], an algorithm for this problem was presented. The algorithm runs sions. In Dekel and Sahni[1983], an algorithm for this problem was presented. The algorithm
on an EREW P-RAM. Then, Bar-On and Vishkin[1985] described a faster, even optimally parallel, algorithm, yet running on a less restricted machine model, the CREW P-RAM. By way the mos algorithm, yet runing matching can be done in $O(\log n)$ time using $O(n / \log n)$ processors.

The bracket matching problem has appeared to be a key problem in parallel parsing. This is because it is the bracket-like construction (embedding) that distinguishes context-free languages from cause it is the bracket-like construction (embedding) that distinguishes context-free languages from
regular ones. A vast range of papers is dedicated to this sole problem of parailel bracket matching. Sarkar and Deo[1988] discuss another optimally parallel CREW P-RAM bracket matching algorithm, which is essentially the same as the one in Mattheyses and Fiduccia[1982]. However, in this rithm, which is essentially the that only $O(n / \log n)$ instead of $n$ processors were needed. See last paper, it was not recognized. The first paper to present an optimally parallel EREW P-RAM also Rytter and Giancarlo[1987]. The first paper to present an optimally parallel EREW P-RAM algorithm for this problem was Tsang et al.[1989]. Later papers, Deo et al.[1990], Prasad and Deo[1991], Chen and Das[1990], and Das et al.[1991] present EREW P-RAM algorithms that are claimed to be simpler, but still optimally parallel. Pitsch and Schðmer[1991] present optimal (averaye case) parallel recognition of bracket languages on hypercubes.

(average case) parallel rim By the existence of optimal EREW P-RAM bracket matching algorithms, it can be

parsing arithmetic expressions can be done optimally in parallel on an EREW P-RAM.

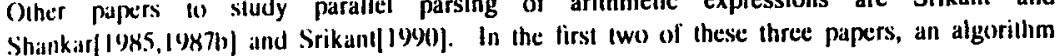

that takes $O\left(\log ^{2} n\right)$ time using $n$ processors is presented. The other one contains an algorithm for mesh, cube and cube-connected cycles parallel computers, resulting in different time bounds. Bracket matching is achieved by sorting. Srikant and Shankar[1987a] present a new way of specifying the syntax of programming languages, called Hierarchical Language Specification. Basically, the regular constructs are separated from the bracket parts and treated differently.

Bracket matching can be used to parse much larger classes of context-free grammars. In Sarkar and Deo[1987], brackets are inserted in the strings of what they call restricted block-structured context-free languages.

Optimally parallel bracket matching algorithms are also used in Oude Luttighuis[1989,1990a, 1991] The first two of these papers deal with parsing regular right-part grammars. The algorithm works for regular right-part grammars that generate a subclass of the LL(1) languages. It constructs the depth-first left-to-right traversal of the parse tree before building the parse tree first. Then, matching the steps down with the steps up in this traversal (using a bracket matching algorithm) yields the parse tree. The third paper uses contexl-free grammars that satisfy the $\operatorname{LE}(p, q)$ constraint. They constitute almost the class of all $L L(k)$ grammars. The algorithm extracts pieces of the parse tree from a precomputed table, taking into consideration a look-back and look-ahead string. One piece corresponds to a series of production applications followed by one shift in the LL algorithm. The $\operatorname{LE}(p, q)$ classes constitute the largest class of CFLs, yet known to be parsable in optimally parallel time.

Bottom-up parsing. The first substantial work to have appeared on parallel parsing is found in Fischer[1975b]. One of the algorithms in this Ph.D. thesis is a parallel parsing method based on LR parsing. It uses a number of (sequential) parsers, all starting at some point in the string. A problem here is the state in which each of the parsers should start. The proposed solution is to maintain a parsing stack for every possible state the (sequential) parser starts in, considering the point at which it starts. When the stack is too small to perform some reduction, the parser merges his stack with the stack of a neighboring parser and dies. Similar ideas appear in Pronina and Trakhtengerts[1974], Mickunas and Schell[1978], Schell[1979] and (very briefly) in Carlisle and Friesen[1985].

In his Ph.D. thesis, Gafter[1990b], the author describes parallel algorithms for incremental lexical, syntactical and semantic analysis. A usual text editor is stipulated to yield a log of edit actions. This $\log$ is used to change the old parse tree. For parsing, an adaptation of Schell[1979]'s LR paralle! parsing algorithm is used. It is recognized that the depth of the parse tree is a bottleneck in speeding up parallel parsing. This depth is especially increased by numerous applications of list productions. The proposed solution to this is to use, instead of left or right recursive productions, rules of the form $A \rightarrow A A$, so that logarithmic depth is achicved. To overcome the extreme ambiguity caused by these rules, the LR algorithm and items are adjusted. A formalism to express the scoping structure of programming languages, that allows for parallefization, called upward remote aggregates, is introduced. Gafter[1987,1990a] can be found in this thesis. Parallel incremental LR parsing is also discussed in Viswanathan and Srikant[1991].

Parallel prefix sum. The parallel prefix sum algorithm can also be used for parsing contextfree grammars, as shown by Skillicorn and Barnard[1989]. This paper uses the parallel prefix sum algorithm to combine sets of stack transitions. These stack transitions are pairs of stack configurations and indicale how the stack changes (during LL(1) parsing) during processing of a 
substring. Stack transition sets corresponding to substrings are pairwisely combined to yield the stack transition set corresponding to the concatenation of the substrings. The claim of the authors sthe ack the used See Oude Luttighuis[1990b] and Hill[1992]. The latter of these discusses an implementation of See Oude Luttighuis[1990b] and Hill[1992]. The latter of these discusses an impleme
the parsing algorithm on a distributed processor array with a two-dimensional topology.

Other papers. In Lampe[1990ab], parallelizing parsing is restricted to grammars that allow to Other papers. Within the grammar, local make parsing decisions on the basis of very local context information. With the grammar, local grammars are distinguished, which have to have this local parsability property (within the overall grammar).

In Loka[1984] and Tseytlin and Yushchenko[1977], one processor starts parsing from left to righ at the beginning of the string, the other starts at the other end, parsing in the opposite direction. Somewhere in the middle, they meet and their parses are combined.

Fischer[1975b] also contains a vector precedence parsing algorithm and a vector algorithm for parsing (and compiling) arithmetic expressions. This last algorithm also appears in Fischer[1975a,1980].

\section{Parallel Context-Free Parsing in Natural Language Processing}

In natural language processing circles there is a wide-spread interest in parallel processing techniques for language analysis. Unlike the compiler construction environment with its generally accepted theories, in natural language processing no generally advocated - and accepted - thecry of the improvement of sped, but it is also the assumption that human sento exploit parallelism for the improvement of speed, but it is also the assumption that human sentence processing is of an inherently parallel nature which makes computer linguists and cognitive scientists tum to parallel approaches for their problems. For these reasons, in natural language processing many kinds of parallel approaches can be distinguished. While some researchers aim prositive language systems. The former cogetween processors that researchers may ulimaty ask for numbers of processors and consections belween processor con that is, an order of $10^{11}$ neurons with $10^{3}-10^{4}$ connections each). They model human language processing with connectionist models and therefore they are interested in massive parallelism, distributed representation of knowledge and low degradation of overall behavior in the face of local errors. We will not discuss Connectionist implementations of conventional approaches to parsing have been proposed. These Connectionist implementations of conventional approaches to parsing have been proposed. These can be seen as boolean circuit implementations of parallel parsing algorithms. For sentences up [1985], Nijholt [1990], Sikkel and Nijholt [1991].

Any system used for understanding natural language sentences needs to distinguish different levels Any sysict the semantic and the the referential level. For each level a different kind of knowledge has to be invoked. Therefore
different tasks can be distinguished: the application of morphological knowledge, the application of lexical knowledge, etc. It is not necessarily the case that application of one type of knowledge is under control of one of the other types of knowledge. The tasks may interact and at times they can be performed simultaneously. Therefore processors which can work in parallel and which can communicate with each other may be assigned to these tasks in order to perform this interplay of multiple sources of knowledge. Hence, this task-oriented paralletism requires smart processors performing specialized functions.

Finally, and independent of a parallel nature that can be recognized in the domain of language processing, since operating in parallel with a collection of processors can achieve substantial speed-ups, designers and those who implement natural language processing systems will consider the application of available parallel processing power for any task or subtask which allows that application. We distinguish two views on this, not necessarily linguistically motivated parallelism. The first view is the programming language point of view, the second is the processor or process point of view. In the first view the starting point is the programming language and the problem of language analysis is programmed using the parallel concepts that are offered by the programming language. For instance, parallel logic programming languages such as GHC (Guarded Horn Clauses), Parlog and Concurrent Prolog invite us to approach the analysis problem from a parallel point of view. Many natural language processing systems based on these parallel logic programming languages have been built. In Matsumoto[1987] and Matsumoto and Sugimura[1987] examples of this approach, using a left-corner method, can be found. In Tanaka and Numazaki[1989] GHC is used to implement a paraliel version of Tomita's generalized LR parsing algorithm. In the papers of Matsumoto the terminal and nonterminal symbols are defined as parallel processes. In the paper of Tanaka and Numazaki each LR-table entry is defined as a process. Similarly, a parallel object-oriented point of view can be advocated, as is done by Yonezawa and Ohsawa[1989]. They use the parallel object-oriented programming language $\mathrm{ABCL} / 1$ to implement a parsing system which is obtained by translating a collection of context-free grammar rules into a configuration of message passing, cooperating units (agents). Each occurrence of a terminal or nontermina symbol in a grammar rule is represented as an agent in the system, the messages that are sent consist of control data or partial parse trees. Designing parsing methods from the point of view of available or desired processor configurations has led to a variety of methods. Attacking the context-free parsing problem with more than one processor almost always means using identica processors, that is, processors that run the same software and compute the same function. In some cases this can amount to having several asynchronous parsers working on the same input string (e.g., Fischer[1975], Loka[1984], Lozinskii and Nirenburg[1986], Sikkel and Lankhorst [1992]). Traditional parsing methods have been re-investigated in crder to see whether thcy can be adapted to a parallel processing view. For the Earley and the Cocke-Younger-Kasami (CYK) parsing methods implementations have been designed for multi-processor shared-memory computers, for pipelines of processors and for arrays of processors. A survey and an explanation of these methods is given in Nijholt $[1989,1990 \mathrm{~b}]$

\section{Complexity of Parallel Context-Free Language Recognition and Parsing}

The main practical reason for introducing parallel algorithms for problems which are not intrinsically parallel is to decrease the processing time needed by sequential algorithms. There is, besides 
this practical motivation, a more theoretical question which motivates investigations after parallel solutions for particular problems. Is it possible to find a parallel solution which is essentially better, as far as processing time is concerned, than sequential solutions for the same problem? Or, stated in a more general way, what kind of operations or problems are intrinsically sequential and which are not? The specific question here is then, can we reduce time complexity by using parallelism in (context-free) language recognition and parsing?

In parallel complexity theory processors are considered as a resource just like time and space in sequential complexity theory. Trends in hardware technology suggest that it becomes more and more reasonable to regard the number of simple processors available for a job as unbounded. As an example of this technology, may serve the Connection Machine which has ten thousands of processors.

For a good parallel algorithm the product of (parallel) time and the number of processors used, will be of the same order of magnitude as the time complexity of a good sequential solution. A algorithm satisfying this is often called optimally parallel.

The theory of context-free parsing has emerged from the practice of making automatic parsers and translators for programming or natural languages. In the area of compiler writing systems, it is sometimes said that if someone writes a grammar for a programming language it is almost LR(1) and with some minor changes it can be made to be LALR(1). This means that such a language can be parsed in linear time by a rather space efficient sequential parsing method. Since the existence of Yace and other compiler generating tools there is hardly any practical motivation within this area of computer science for using parallelism. The situation seems to be different in the area of natural languge processing where traditionally one is more interested in grammars which are not natural language proces for recognition and parsing of general context-free languages are often used and studied in this field. Valiant's variant of the CYK-method which runs in time $O\left(n^{2.81}\right)$ is the fastest sequential parsing algorithm for general CFLs. Earley's method takes time $O\left(n^{3}\right)$, but it has the advantage that it does not need a grammar in Chomsky Normal Form. Particularly, if one is dealing with parsing and translating general CFLs one may have a practical interest for speeding up processing time by using parallel algorithms and parallel architectures. About the same time as Valiant came up with his sequential algorithm, Kosaraju[1975] showed that the CYK-method can be used to recognize general CFL $s$ in $O(n)$ time on a two-dimensional array of processors. A lot of papers are devoted to parallel versions and implementations of Earley's method. These show that this can to done in linear time by using $O\left(n^{2}\right)$ processors and $O\left(n^{2}\right)$ of total space. Recently, de Vreught and Honig[1989] came up with a general recognition method which resembles the tabular Earley algorithm. Instead of one, as usual, items have two dots, as in Bossi et al.[1983]. The method works bottom-up and doesn't use the predictor operation. A parallel algorithm for a P-RAM shows that this method has a parallel solution with complexity properties like those for Earley's method and the CYK-method.

Confronied with several parallel algorithms for different particular architectures the problem arises how we can compare them. Suppose we have a polynomial parallel time solution on a particular model of parallel computations. Does this mean that we also have a polynomial solution on any "Wher pardillel macchine model?" How are different moxlels related to each other? There is a large variely of menkels firr sequential computation, all of which can be used as defining the class of cimputable functions. Best known are the Turing machines (TM) and the random access machines (RAM). Must of these models have a measure for computation resources (like time and space) such that whenever a machine $M$ in class $A$ runs in time $T$ and space $S$, then there is a simulating machine $M^{\prime}$ in class $B$, such that the time used for the simulation of $M$ is polynomially related to $T$ and the space used by $M^{\prime}$ is of the same order of magnitude as $S$.

For a "reasonable" model of sequential computation there are some complexity classes, the definition of which is invariant. under the specific model used. These classes form the following hierarchy of sequential complexity classes.

\section{LOGSPACE $\subset$ NLOGSPACE $\subset P \subset N P \subset$ PSPACE $=$ NPSPACE $\subset$ EXPTIME $\subset$ NEXPTIME}

It is open whether the inclusions are Icany proper or not. The equality in this sequence is the well-known theorem of Savitch.

For parallel computations there is even a greater variety of "reasonable" machine models. A model is called "reasonable" with respect to parallel computation if it satisfies the Parallel Complexity Thesis. This thesis states that parallel time is polynomially related on all reasonable models. Moreover time on such a model should be polynomially equivalent to (log-cost) space on a sequential model. This means that the following equalities should hold:

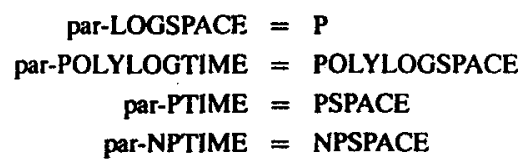

and so as a consequence of Savitch theorem par-PTIME = par-NPTIME. Thus deterministic parallelism can give more than polynomial speed-up if PTIME $=$ PSPACE.

An often used model of parallelism is the CREW or P-RAM introduced in Fortune and Wyllie[1978] or variants of it like the SIMDAG model of Goldschlager or the CRCR or W-RAM in which multiple writes into the same global memory location are allowed. Other "reasonable" models are combinatorial circuits (cf. Borodin[1977] and Ruzzo[1981]) and alternating Turing machines (cf. Ruzzo[1979] and lbarra et al.[1988]).

As far as the complexity of context-free recognition and parsing is concerned the situation is as follows. An obvious lower-bound for sequential time is linear. The best known algorithms have more than quadratic time complexity (Earley's algorithm, CYK-algorithm). Each CFL can be recognized using polynomial time and $n^{2}$ space. Some subclasses of DCFL (the class of deterministic context-free languages) are known to be in DSPACE $(\log n)$, bracket languages (Mehlhorn, Information Processing Letters 5(6), 1976) and input-driven languages (Von Braunmül and Verbeek, Proceedings Fundamentals of Computation Theory 1983, 40-51). See also Rytter[1986a]. For deterministic CFLs, Cook[1979] was the first in which is presented a polynomial time and simultaneous $\log ^{2} n$ space recognition algorithm. It is not known whether all DCFLs are recognizable in $\log n$ space. It is also not known whether all CFLs are in the class SC (simultaneously polynomial time and poly-log space). Ruzzo $[1979,1981]$ has shown that they are in $\mathrm{NC}^{2}$. In Larmore and Ryiter[1992] sublinear parallel time algorithms are presented for the recognition of linear, unambiguous, and deterministic conlext-free languages are presented. 'These results imply an improvement of the known $\mathrm{NC}$ algurithms for dynamic programming.

$\mathrm{NC}^{k}$ is the class of languages recognizable in $O\left(\log ^{k} n\right)$ time by a polynomial number of processors (see Pippenger[1979] and Ruzzo[1981]). 
There are sevcral methods for proving upper-bounds for language recognition. One method is simulation of a (deterministic) PDA. Cook[1979] gives a simulation of a DPDA on a DTM within simulation of a (deterministic) PDA. Cook[1979] gives a simulation of a DPDA on a DTM within result by giving an algorithm that works on a DTM in time $O\left(n^{2} / \log ^{2} n\right)$ and space $\log ^{2} n$. In Von Braunmühl, Cook, Mehl horn and Verbeek[1983] a new algorithm is presented for DPDA simulation on a DTM within the same time and space bounds. The idea is to simulate a divide-and-conquer algorithm for checking whether $C_{\text {insitial }} t^{*} C_{\text {eccept }}$ on the DTM.

A parallel simulation of a DPDA is also presented in Reif[1982] which results in an $O(\log n)$ A parallel simulation of a DPDA is also presented in Reif[1982] which results in an $O(\log n)$
algorithm for recognition of deterministic CFLs. This method is not very practical. Optimal parallel recognition methods are known for bracket languages and input-driven languages (Gibbons and Rytter[1989]) and for Dyck-languages (Mattheyses and Fiduccia[1982]) and regular languages. In Ibarra[1988] it is shown that the classes of one-sided Dyck languages, bracket languages and a subclass of the linear languages are in $\mathrm{NC}^{1}$.

Optimal parallel parsing methods are known for bracket languages (Rytter and Giancarlo[1987]) Dyck-languages (Mattheyses and Fiduccia[1982]) and for DFLR(1) and LE $(p, q)$ languages, both subclasses of the class of $L L(1)$ languages (Oude Luttighuis [1989,1990a]). In all these papers an optimal parallel method for matching of parentheses like that presented in Bar-On and Vishkin[1985] is essential.

Some methods for general CFL recognition which lead to $\log n$ parallel algorithms are based on the Some methods for general CFL recognition which lead to log $n$ parallel algorithms are based on the
following idea. Given a string $a_{0} \ldots a_{n}$ and a context free grammar $G$. The problem of recognition of $L(G)$ can be formulated as deriving a statement in a theory. There are $n$ axioms $a_{i}$, denoted by $\vdash\left(i, a_{i}, i+1\right)$ and the rules are given by the production rules of $G$. If $A \rightarrow B C D$ is a production then, we have $\langle i, B, j\rangle,\langle j, C, k\rangle,\langle k, D, p\rangle \vdash\langle i, A, p\rangle$ as a derivation rule in our theory. Since we only have to look al rules with $i \leq j \leq k \leq p$ we have a polynomial number of rules. We have to prove that $\langle 0, S, n\rangle$ which denotes that $a_{0} \ldots a_{n}$ can be derived from $S$, the start symbol of $G$. For grammars in CNF, this view is worked out in Brent and Goldschlager[1985] and in Rytter[1987b]. grammars in CNF, this view is worked out in Brent and Goldschlager[1985] and in Rytter[1987b].
Rytter shows by using this method that unambiguous CFLs can be recognized in $O(\log n)$ time on a P-RAM time by using $O\left(n^{6}\right)$ processors. Each triple $\langle i ; A, j\rangle$ is assigned a processor. Bren and Goldschlager use a similar method for the SIMDAG model and show that any CFL can be recognized and parsed within the same complexity bounds, i.e. by $O\left(n^{6}\right)$ processors in $O(\log n)$ time. The amount of processors seems to be too large to be practical.

Some authors do not carefully distinguish recognition and parsing. In Rytter[1987a] it is shown that when we consider parallel time then parsing is indeed not harder than recognition, contrary to the sequential case. See also Oude Luttighuis[1991] in which it is shown that on a P-RAM ther is no essential difference in complexities between parallel parsers yielding derivations and those yielding parse trees. A quit assumption made in all the literature on parallel parsing.

Another bibliography on the theory of parallel recognition and parsing is de Vreught![1991]. Here you can also find a short overview of work in this area.

\section{Parallel Compilation}

Although this bibliography mainly concentrates on parallel parsing, if also includes papers on parallef compilation in general. Cumpilers are amung the tools most heavily used by programmers. Therefore, the speed of compilation is directly related to programmer productivity. One way to speed up compilation is to exploit parallelism. Other techniques to reduce compile time are incremental compilation and separate compilation of program modules. Parallel compilation can make both of these techniques faster. As parallel processing technology advances, concurrency becomes an attractive vehicle by which compilation speed may be increased.

Theoretical research in the field of parallel compilation has dealt mainly with its best understood area - parsing regular and context-free grammars (e.g., Mickunas and Schell[1978]). The first significant theoretical investigation into other phases of compilation has been the work of Schell[1979]. In addition to lexical and syntactic analysis, his thesis examined semantic analysis based on parallel attribute evaluation. Any practical investigations in concurrent compilation have naturally been constrained to the multiprocessing hardware available at the time. Early efforts were aimed at vector processors, while more recent research has been directed towards loosely coupled distributed systems and more tightly coupled MIMD multiprocessors.

The first practical attempts to parallelize compilation date back to the early 1970s. Most of these efforts were restricted to making certain phases of compilation concurrent for a specitic language (i.e., FORTRAN, APL) on vector processors (e.g., CDCSTAR-100), which were the only multiprocessors available at the time. Lincoln[1970] showed how parallel techniques for lexical analysis and parsing of FORTRAN programs may be expressed by APL vector operations. Donegan and Katzke[1975] elaborated Lincoln's theme and Krohn[1975] extended it to code generation. Similar attempts to apply vector processing techniques to compilation were reported by Ellis[1971] and Zosel[1973]. See also House[1981].

Local area network technology later in the 1970 s, offered prospects for more coarsely grained parallel compiler designs. The earlier efforts which utilized this kind of distributed processing concentrated on a pipelined approach to concurrent compiling. Pipelining is a common technique to exploit concurrency in tasks that can be divided into a series of stages. The stages are cascaded so that the output of one stage is the input for the next. Some form of buffering is needed between each pair of stages to facilitate the transfer of information from one stage to the next. If each stage runs concurrently, the maximum speedup is equal to the number of stages.

The easiest way in which to transform a sequential compiler into a concurrent compiler is to run the compiler phases as separate processes in a pipeline, and to have them communicate through shared data structures. This approach has two limitations. First, the degree of concurrency is limited by the number of stages in the pipeline, which results in the utilization of only a fixed number of processors, thus potentially leaving available processors idle. Second, the overall speed of compilation is constrained by the speed of the slowest pipeline stage. Many compilers spend a large fraction of their execution time in one or two phases. Much of the effort to implement an efficient pipeline may go to balancing the different stages. Consequently, pipelining the phases on a multi-processor will generally yield a less than ideal speedup.

Designs for pipelined compilers have been presented in several papers. Baner and 1:llis: [977] discussed a graph model based on a generalized Petri Net to describe the control and data flow of an existing compiler. The modeled version of the compiler was modified so that its sequential structure cuuld be easily transformed into a pipeline of processes. Miller and LeBlanc[1982] compared for a subset of Pascal a sequential single-pass compiler with a distributed version. The stages in their pipeline consisted of a lexical analyzer, a syntactic annlyzer and a semantic analyzer/code generator which emitted intermediate code. Huen et al. [ [ 977$]$ described the design of a pipelined DYNAMO compiler which ran on a network computer. The compiler produced parallel code segments for the same network computer. The object code was autematically partitiened into) 
clusters so that the clusters could execute in parallel on the individual computers in the network computer. See also Christopher et al.[1981]

Instead of determining which phases of the compilation process can be carried out concurrently, one may construct a compiler which splits the source program into segments which are then compiled concurrently. These segments would typically be well-defined syntactic constructs, such as procedure bodies or statements lists. From the point of view of semantic analysis, the source as procedure bodies or statements lists. From the point of view of semantic analysis, the source
code must be partitioned in such a way as to minimize the amount of inter-process communication. Unlike the pipelined scheme, this approach does not innerently limit the amount of concurrency which can be exploited using the underlying hardware - that is, the amount of concurrency which exists in the compiler is determined to a greater extent by the characteristics of the source program being compiled and the underlying hardware, rather than by the structure of the compiler: These efforts have been targeted at both loosely coupled uniprocessor networks and more recently developed shared MIMD machines.

Lipkie[1979] was probably the first to suggest a combination of pipelining and concurrent processing of program segments. Lipkie proposed the design of a parallel compiler which broke the source code at procedure boundaries. The source code division would occur during parsing, after a sequential prescanning phase. Each procedure would be processed concurrently with the others, with the resultant code merged at the end. For each procedure definition a pipeline would be instantiated. Lipkie's thesis did not report on any implementation.

$\checkmark$ A related, but more general approach, can be found in the thesis of Messerer[1982]. The source program is subdivided into fragments. During the combined lexical and structural analysis of any fragment a partial structure graph is built whose nodes represent blocks, procedures and control structures. From the partial graphs a total graph is composed which reflects the structure of the entire program. This graph indicates how the program can be subdivided into blocks and control structures which can be analyzed in parallel. Experimental results are not reported.

Frankel[1983] extended a one-pass recursive descent Pascal compiler to translate procedures concurrently. Whenever a compiler instance encounters a child scope while compiling a parent scope, it creates a new instance to compile the child scope and skips to the end of the child scope by matching delimiters.

Vandevoorde[1988ab] constructed a concurrent $C$ compiler that runs on a tightly coupled multiprocessor. The compiler consists of a two-stage pipeline. The first stage performs extended lexical analysis for the second stage, which does the parsing, semantic analysis, and code generation. The second stage processes units of the source program concurrently. The division of the source code is accomplished in a manner similar to that of Frankel's compiler. However, Vandevoorde's compiler incorporates a finer grain of parallelism. Large statements are broken into smaller units which are processed concurrently through semantic analysis and code generation. Vandevoorde's compiler differs from previous concurrent compilers in several ways. First, it extends the role of the scanner to include matching delimiters for the parser. This allows the parser to skip to the end of a child scope guickly. Second, Vandevoorde's compiler uses finer grains of parallelism than have been used on distributed systems. Previous compilers have processed only procedures in parallel. Vandevoorde's compiler processes units as small as a simple statement concurrently. Finally, it runs on a tightly coupled multi-processor. Since such computers have only recently become widely available, most previous parallel compilers have been built on top of distributed workstations. Seshadri et al.[1988abc,1991], Junkin and Wortman[1990], Wortman[1990], and Wortman and Junkin[1992] used a similar approach to build parallel Modula-2+ compilers to run on a dis- tributed system. They do not restrict themselves to the parallel compilation of statements, but they investigate the concurrent processing of declarations as weil. The latter introduces what they call the "Doesn't Know Yet" problem: the compiler may attempt to access information introduced by a declaration before it has been processed. Other implementations of parallel compilers are reported by Brezany[1986] and Gross et al.:[1989].

Attribute grammars have proved to be a useful formalism for specifying the syntax and the semantics of programming languages, as well as for implementing editors, compilers, translato writing systems and compiler generators. For a given syntax tree the evaluation order of its attributes is only restricted by the partial order induced by its dependency graph. A sequential evaluator completely serializes this order. Parallel evaluators are based on partial orders only. The thesis of Schell[1979] includes a chapter devoted to parallel tree-walk evaluation. His work is of a design nature, and did not result in any implementation. Practical experiments were reported by Jourdan and Marmol, Lampe, Kaplan and Kaiser, Boehm and Zwaenepoel, and Kuiper and Dijkstra. Jourdan[1991] and Marmol[1990] independently rediscovered the method of Schell and reported a simple and efficient implementation. Lampe[1988,1990)c] reported concurrent attribute evaluation based on an event-driven control scheme. Kaplan and Kaiser[1986] presented a model for distributed program editing. Their algorithm for incremental attribute evaluation allows multiple asynchronous edits on program modules that are distributed across a number of workstations connected by a high speed network. Boehm and Zwaenepoel[1987] developed a compiler-generator which produces a sequential parser and a number of parallel attribute evaluators from a single attribute grammar specification. The parser builds the syntax tree, divides it into subtrees, and sends them to the attribute evaluators. The attribute evaluators then proceed with the actual translation by evaluating attributes belonging to the symbols in their subtree, transmitting values of shared attributes as necessary. For efficiency, both static and dynamic attribute evaluators are used. Static evaluation is used to evaluate all attributes local to a subtree. Dynamic evaluation is applied for attributes which are shared between subtrees. Boehm and Zwaenepoel used this method to generate a compiler for a large Pascal subset, which runs on a collection of up to 6 SUN-2 workstations connected by an Ethernet. Alblas[1990] proposes a combination of Boehm and Zwaenepoel's scheme with that of Kaplan and Kaiser for the incremental reevaluation of attributes after a tree transformation. He also formulates criteria based on safe approximations of a consistently attributed tree which permit a delay in calling the reevaluator until after severa tree modifications. Thus, different reevaluations and different tree transformations may occu asynchronously and concurrently in different regions. The only constraint is that each region is either in its transformation phase or in its reevaluation phase.

A significant theoretical investigation into parallel attribuie evaluation is the work of Kuiper[1989] His thesis discusses various methods for distributing attribute computations over processes. I includes an algorithm that analyses attribute grammars in order to statically detcet independent computations. The analysis is static, in the sense that it is performed as part of the construction of the attribute evaluator (see also Kuiper and Swierstra[1990]). Kuiper also presents a method that increases the amount of potential parallelism by removing attribute dependencies and thus cutting linear chains at the cost of adding extra attributes (see also Kuiper[1988]). Experiments with attribute evaluation on a network of Iransputers are reported by Kuiper and Dijkstra| (984)|. Klein [1990,1991,1992] describes an approach based on the notion of graph of each production is statically partitioned into segments, with the condition that each iwo attributes of a nonterminal are always in the same segment in every production or in distinct 
segments. At run-lime the various segments are vertically melted whenever they have at least one common attribute instance. This leads to a partition of the global dependency graph into a number of segments. In each segment the dependencies are linearized, but the evaluation of the instances in different segments can proceed concurrently, with synchronization points defined by cross-segmen dependencies. Criteria are presented that allow to statically precompute the evaluation order in the segments. These criteria define a new class of parallel ordered attribute grammars. The construction of parallel visit sequences is performed by a polynomial-time algorithm.

Klein and Koskimies $[1989,1990,1991]$ present a syntax-directed compilation scheme in which a parser runs in parallel with a set of parallel processes that carry out semantic computation To establish a pipeline-type connection between the sequential parser and the set of paralle semantic processes, a tree-structured pipeline buffer is developed, allowing several waiting points for arbitrary many semantic processes. The method can be applied to a variety of parallel semantic evaluation strategies. The only restriction is that semantic analysis works in a syntax-directed way. Parallel one-pass evaluation was the original motivation for this work.

Klaiber and Gokhale[1992] report experiments on parallel attribute evaluation for absolutely noncircular attribute grammars. Non-interdependent attributes instances can be evaluated in parallel. To avoid processes with low load, weights are assigned to attributes, thus providing a means by which it can be decided at evaluation time whether or not to fork a subprocess.

Zaring $|(\mathcal{W})|$ gives a quite thorough analysis of the construction and implementation of parallet cxhaustinc and incremental visit-sequence-based evaluaturs. He considers both asynchronous

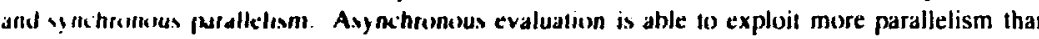

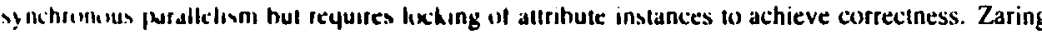
preselts several algorrthms lest the different parallel evaluation moxkels.

A survey of parallet ittribute evaluation methods can be found in Jourdan(19911. This paper reviews and compares the various methods that have appeared in the literature for both exhaustive and incremental altribute evaluation on both tightly-coupled (shared-memory) and loosely-coupled (distributed) architectures.

Many compilers spend a large fraction of their execution time in back-end phases such as instruction selection, register allocation, and optimization. This means that the parallelization of these phases is imperative to fast compiler operation. Parallelization of these phases should therefore be explored in the near future. Zobel[1990] states that the parallelization of machine-independen global optimization should be the core area of investigation. A basic component of optimization, which may be carried out many times during the compilation of a program, is data-flow analysis. Results on the parallelization of data-flow analysis are reported by Gupta et al.[1990] and Lee et al. [1990,1991].

Efforts in concurrent compiling that have thus far not been mentioned are the work of Banatre et al., Deth, Dowsing and Sanderson, Katseff, and Alblas. Banatre et al.[1979] consider an event-driven technique for semantic analysis. Deth[1984] describes an analyzer which maps a program onto a graph of semantic routines. It is shown how this graph can be evaluated in parallel, and also how the sequential evaluation of the semantic functions can be parallelized. Dowsing and Sanderson[1986] investigate the use of Path Pascal for implementing an assembler on a distributed computer system. They report experiments using simulated concurrency on single processor computer. Katseff[1988] applies the technique of data partitioning to implement a parallel assembler. Alblas [1992] investigates the parallelization of a parser generator.

In the lileralure a large number of papers are devoled to the parallel execution of programs, and in particular to the parallel evaluation of arithmetic expressions. These papers are not included in this bibliography. Also not included in this bibliography are papers on compiler optimizations for supercomputers.

Another survey of parallel compilation can be found in Skillicorn and Barnard[1990a]. See also Skillicorn and Barnard[1990b]

\section{Bibliography}

Akker, H.J.A. op den, H. Alblas, A. Nibholt, and P.H.W.M. Oude Luttighuis [1992]. An annotated bibliography on parallel parsing: updated version. Memoranda Informatica INF 92-84, Department of Computer Science, University of Twente, Enschede, The Netherlands, December 1992.

Alblas, H. [1990]. Concurrent incremental attribute evaluation. Proceedings Internutional Workshop on Attribute Grammars and their Applications (WAGA), Paris, September 19-21, 1990. Lecture Notes in Computer Science 461, Springer Verlag, Berlin, 1990, 343-358.

Alblas, H. [1992]. A blueprint for a parallel parser generator. Memoranda Informatica INF 92-65, Department of Computer Science, University of Twente, Enschede, The Netherlands, October 1992.

André, F., J.P. Banâtre, and J.P. Routeau [1981]. A multi-processor approach to compile-time symbol resolution. ACM Transactions on Programming Languages and Systems 3(1), 1981, 11-23. Asthagiri, C.R., and J.L. Potter [1992]. Associative parallel lexing. Proceedings of the 6 th International Parallel Processing Symposium, Beverly Hills, CA, USA, March 1992, 466-469. Babichev, A.V. [1982]. Some theoretical aspects of parallel parsing. Cybernetics 18(2), 1982, 189-195.

Baccelli, F., and T. Fleury [1982]. On parsing arithmetic expressions in a multi-processing environment. Acta Informatica 17, 1982, 287-310.

Baccelli, F., and P. Mussi [1986]. An asynchronous parallel interpreter for arithmetic expressions and its evaluation. IEEE Transactions on Computers 35(3), 1986, 245-256.

Baer, J.L., and C.S. Ellis [1977]. Model, design, and evaluation of a compiler for a parallel processing environment. IEEE Transactions on Software Engineering SE-3(6), 1977, 394-405.

Banâtre, J.P., J.P. Routeau, and L. Trilling [1979]. An event-driven compiling lechnique Communications of the ACM 22, 1979, 34-42.

Bar-On, I., and U. Vishkin [1985]. Optimal parallel generation of a computation tree form. ACM Transactions on Programming Languages and Systems 7(2), 1985, 348-357.

Boehm, H.-J., and W. Zwaenepoel [1987]. Parallel attribute grammar evaluation. Proceedings 7th International Conference on Distributed Computing Systems, R. Popescu-Zeletin, G. Le Lam K.H. Kim (eds), Berlin, Germany, 1987, 347-354.

Borodin, A. [1977]. On relating time and space to size and depth. SLAM Journal on Computing 6(4), 1977, 733-744.

Bessi, A., N. Cocco, and L. Colussi [1983]. A divide-and-conquer approach lo generul comlextfree parsing. Information Processing Letters 16, 1983, 203-208.

Braunmühl, B. von, S. Cook, K. Mehlhorn, and R. Verbeek [1983]. The recognition of deterministic CFLs in small time and space. Information and Control 56, 1983, 34-51. 
Brent, R.P., and L.M. Goldschlager [1984]. A parallel algorithm for context-free par: Australian Computer Science Communications 6(1), 1984, 7.1-7.10.

Brezany, P. [1986]. Parallel compilation on a multiproces sor system. Proceedings of CON 86, Conference on Algorithms and Hardware for Parallel Processing, Aachen, September 17 1986, Lecture Notes in Computer Science 237, Springer Verlag, Berlin, Germany, 1986, 279Carlisle, W.H., and D.K. Friesen [1985]. Parallel parsing using Ada. Proceedings 3rd An National Conference on Ada Technology, March 1985, 103-106.

Chang, N.-S., and K.S. Fu [1979]. Parallel parsing of tree languages for syntactic pal recognition. Pattern Recognition 11, 1979, 213-222.

Chang, J.H., O.H. Ibarra, and M.A. Palis [1987]. Parallel parsing on a one-way array of state machines. IEEE Transactions on Computers 36, 1987, 64-75.

Chen, C.C.-Y., and S.K. Das [1990]. A cost-optimal parallel algorithm for the parentt matching problem on an EREW P-RAM. Technical Report N-90-005, Department of Com Science, University of North Texas, Denton, TX, USA, May 1990.

Chiang, Y.T., and K.S. Fu [1982]. A VLSI architecture for fast context-free language recogni Proceedings of the Third International Conference on Distributed Computer Systems, 1982, 869.

Chiang, Y.T., and K.S. Fu [1984]. Parallel parsing algorithms and VLSI implementations syntactic pattern recognition. IEEE Transactions on Pattern Analysis and Machine Intellige PAMI-6(3), 1984, 302-314.

Chrisțopher, T., O. El-Dessouki, M. Evens, H. Harr, H. Klawans, P. Krystosek, R. chandani, and Y. Tarhan [1981]. SALAD - A distributed compiler for distributed syst Proceedings of the International Conference on Parallel Processing, 1981, 50-57.

Proceedings of the International Conference on Parallel Processing, 1981, 50-57.
Chu, K.H., and K.S. Fu [1982]. VLSI architectures for high-speed recognition of contextlanguages and finite-state languages. Proceedings of the Ninth Annual Symposium on Comp Archilectures, SIGARCH Newsletter 10(3), 1982, 43-49.

Chytil, M.P., and B. Monien [1988]. Caterpillars and context-free languages. Bericht $\mathrm{Nr}$. Universität Paderborn, 1988.

Cohen, J., T. Hickey, and J. Katcoff [1982]. Upperbounds for speed-up in parallel pars Journal of the ACM 29, 1982, 408-428.

Cohen, J., and S. Kolodner [1985]. Estimating speed-up in parallel parsing. IEEE Transact. on Software Engineering 11, 1985, 114-124.

Cook, S.A. [1979]. Deterministic CFLs are accepted simultaneously in polynomial time and squared space. Proceedings of the 11th Annual ACM Symposium Theory of Computing, 1! 338-345.

Crochemore, M., B. Monien, and W. Rytter [1991]. On the parallel recognition of unambig contexl-free languages (note). Theoretical Computer Science 81(2), 1991, 311-316.

Das, S.K., C.C.-Y. Chen, G. Lewis, and S.K. Prasad [1991]. Some fast parallel algorithms parentheses matching. Proceedings of the International Conference on Advances in Compu and Information (ICCI'91), Ottawa, Canada, May 1991, 443-454.

Dekel, E., and S. Sahni [1983]. Parallel generation of postfix and tree forms. ACM Transacti on Programming Languages and Systems S(3), 1983, 300-317.

Deo, N., S.K. Prasad, and D. Sarkar [1990]. Some fast P-RAM algorithms for matcl parcnitheses. Proxeedings of the Workshop on Parallel Compilation, Kingston, Ontario, Can May (WM).
Deth, R. [1984]. Paraliele Bearbeitung Semantischer Routinen im System RUEGEN. Forschungsbericht WPU-PS-07, Universitäût Rostock, Germany, 1984.

Devos, M., G. Adriaens, and Y.D. Willems [1988]. The parallel expert parser (PEP): a thoroughly revised descendent of the word expert parser (WEP). 12th Internutional Conference on Computational Linguistics (COLING'88), Budapest, Hungary, 1988, 142-147.

Donegan, M.K., and S.W. Katzke [1975]. Lexical analysis and parsing techniques for vector machines. Proceedings of the Conference on Programming Languages and Compilers for Paralle and Vector Machines. SIGPLAN Notices 10(3), 1975, 138-145

Dowsing, R.D., and M.T. Sanderson [1986]. W-iting concurrent assemblers - a case study in Path Pascal. Software Practice and Experience 16, 1986, 1117-1135.

Dymond, P.W., and W.L. Ruzzo [1986]. Parallel RAMs with owned global memory and deterministic context-free language recognition. Automata, Languages, and Programming, L. Kott (ed.), Lecture Notes in Computer Science 226, Springer Verlag, Berlin, Germany, 1986, 95-104. Ellis, C.A. [1971]. Parallel compiling techniques. Proceedings of the Annual Conference of the ACM, 1971, 508-519.

Fanty, M.A. [1986]. Context-free parsing with connectionist networks, Proceedings Conference Neural Networks for Computing, Snowbird, UT, 1986, J.S. Denker (ed.), AIP Conference Proceedings 151, American Institute of Physics, 140-145.

Fischer, C.N. [1975]. Parsing context-free languages in parallel environments. Ph.D. Thesis, Technical Report 75-237, Department of Computer Science, Cornell University, Ithaca, NY, USA, 1975.

Fischer, C.N. [1980]. On parsing and compiling arithmetic expressions on vector computers. $A C M$ Transactions on Programming Languages and Systems 2, 1980, 203-224.

Fleury, T. [1983]. Compilation parallele d'un langage à structure de bloc. Thèse de Doctorat Ingénieur, in French, Université Paris-Sud, Paris, France, 1983.

Floyd, R.W., and J.D. Ullman [1980]. The compilation of regular expressions into integrated circuits. Proceedings of the 21st Annual Symposium on Foundations of Computer Science, IEEE Computer Society, 1980, 260-269.

Fortune, S., and J. Wyllie [1978]. Parallelism in random acces machines. Proceedings of the 10th ACM Symposium on the Theory of Computation, 1978, 114-118.

Foster, M.J., and H.T. Kung [1981]. Recognize regular languages with programmable buildingblocks. In: VLSI 81: Very Large Scale Integration, J.P. Gray (ed.), Academic Press, London, England, 1981, 75-84.

Frankel, J. [1983]. The architecture of closely-coupled distributed computers and their language processors. Ph.D. Thesis, Harvard University, Cambridge, USA, 1983

Fusaoka, A., and M. Hirayama [1982]. Compiler chip: A hardware implementation of compiler. Proceedings of Symposium on Architectural Support for Programming Languages and Operating Systems, 1982, 92-95.

Gafter, N.M. [1987]. Algorithms and data structures for parallel incremental parsing. Proceedings of the 1987 International Conference on Parallel Processing, August 1987, 577-584.

Gafter, N.M. [1990a]. On the complexity of parallel compilation. Proceedings of the Workshop on Parallel Compilation, Kingston, Ontario, Canada, May 1990.

Gafter, N.M. [1990b]. Parallel incremental compilation. Ph.D. Thesis, Technical Report TR 349, Deparıment of Computer Science, University of Rochester, Rochester, NY, USA, June 1990. 
Gibbons, A., and W. Rytter [1988]. Efficient Parallel Alonrithms. Cambridge University Press, Cambridge, MA, USA, 1988.

Gibbons, A., and W. Rytter [1989]. Optimal parallel algorithms for dynamic expression evaluation and context-free recognition. Information and Computation 81, 1989, 32-45.

Graham, S.I. and MA. Harrison [1976]. Parsing of general context-free languages. In: Advances in Computers, Vol. 14, M. Yovits and M. Rubinoff (eds.), Academic Press, New Yotk, NY, USA, 1976, 77-185.

Graham, S.L., M.A. Harrison, and W.L. Ruzzo [1980]. An improved context-free recognizer, ACM Transactions on Programming Languages and Systems 2(3), 1980, 415-462.

Grishman, R. and M. Chitrao [1988]. Evaluation of a parallel chart parser. Second Conference on Applied Natural Language Processing, Association for Computational Linguistics, 9-12 February 1988, 71-76.

Gross, T., A. Zobel, and M. Zolg [1989]. Parallel compilation for a parallel machine. Proceedings of the SIGPLAN'89 Conference on Programming Language Design and Implementation, SIGPLAN Notices 24(7), 1989, 91-100.

Guibas, LJ., H.T. Kung, and C.D. Thompson [1979]. Direct VLSI implementation of combinatorial algorithms. Proceedings of the Conference on VLSI, Caltech, January 1979, 509-526.

Gupta, R., L. Pollock, M.L. Soffa [1990]. Parallelizing data-flow analysis. Proceedings Workshop on Parallel Compilation, Kingston, Ontario, Canada, May 6-8, 1990.

Has, A. [1987]. Parallel parsing for unification grammars. Proceedings of $I J C A I-87,615-618$. Hill, J.M.D. [1992]. Parallel lexical analysis and parsing on the AMT distributed array processor. Parallel Computing, 18, 1992, 699-714.

Hillis, W.D., and G.L. Steele [1980]. Parsing a regular language. Section in: Data parallel algorithms. Communications of the ACM 29(12), 1986,1170-1183.

House, S.R. [1981]. Compiling in parallel. Proceedings of CONPAR'81, Conference on Analysing Problem Classes and Programming for Parallel Computing, Nurnberg, Germany, June 10-12, 1981, Lecture Notes in Computer Science 111, Springer Verlag, Berlin, Germany, 1981, 298-313. Huang, X.M. and L. Guthrie [1985]. Parsing in parallel. MCCS-85-40, New Mexico State University, Las Cruces, NM, USA, 1985.

Huen, W., O. El-Dessoukt, E. Huske, and M. Evens [1977]. A pipelined DYNAMO compiler. Proceedings of the International Conference on Parallel Processing, 1977, 57-66.

Ibarra, O.H., M.A. Palis, and S.M. Kim [1985]. Fast parallel language recognition by cellular automata. Theoretical Computer Science 41, 1985, 231-246.

Ibarra, O.H., T. Jiang, and B. Ravikumar [1988]. Some subclasses of context-free languages in $\mathrm{NC}^{1}$. Information Processing Letters 29, 1988, 111-117.

Ibarra, O.H., and M.A. Palis [1990]. An efficient all-parses systolic algorithm for general context-free parsing. International Journal Parallel Programming 19(4), 1990, 295-331.

lbarra, O.H., T.-C. Pong, and S.M. Sohn [1991]. Parallel recognition and parsing on the hypercube. IEEE Transactions on Computers 40(6), 1991, 764-770.

Janickd $\mathbf{R}$ and $T$. Móldner. A simple realization of a parallel device recognizing regularly defined trace languages. Proceedings of the 18th ACM Annual Conference on Computer Science. Washington, DC, USA, February 1990, 145-153.

Janssen, W., M. Poel, K. Sikkel, and J. Zwlers [1992]. The Primordial Soup Algorithm. Proceedings of the 14th International Conference on Computational Linguistics (COLING'92), Nantes, France, 373-379.
Jebelean, T. [1989]. Language recognition by nonpropagating cellular automata. Report of the University of Timişoara, Hungary, 1990.

Jebelean, T. [1990]. Language recognition by nonpropagating cellular automata. Report 90-73, Faculty of Technical Mathematics and Informatics, Delf University of Technology, Delft, The Netherlands, 1990.

Jourdan, M. [1991]. A survey of parallel attribute evaluation methods. Proceedings International Summer School on Attribute Grammars, Applications and Systems, H. Alblas and B. Melichar (eds.), Lecture Notes in Computer Science 545, Springer Verlag, Berlin, Germany, 1991, 234-255. Junkin, M.D., and D.B. Wortman [1990]. The implementation of a concurrent compiler. Technical Report CSRI-235; Computer Systems Research Institute, University of Toronto, Toronto Ontario, Canada, December 1990.

Kaldewaij, A and G. Zwaan [1990]. A systolic design for acceptors of regular languages. Science of Computer Programming 15, 1990, 171-183.

Kaplan, S.M., and G.E. Kalser [1986]. Incremental attribute evaluation in distributed languagebased environments. Proceedings of the Fifth Annual ACM Symposium on the Principles of Distributed Computing, 1986, 121-131.

Katseff, H.P. [1988]. Using data partitioning to implement a parallel assembler. Proceedings ACM/SIGPLAN PPEALS 1988 Symposium on Parallel Programming: Experience with Applications, Languages, and Systems, July 1988, 66-76.

Khanna, S., and A. Ghafoor [1990]. A data partitioning technique for parallel compilation. Proceedings of the Workshop on Parallel Compilation, Kingston, Ontario, Canada, May 1990.

Kindervater, G.A.P., and J.K. Lenstra [1985]. An introduction to parallelism in combinatorial optimization. In: Parallel Computers and Computations, J.K. van Leeuwen and J.K. Lenstra (eds.), CWI-Syllabus 9, Centre for Mathematics and Computer Science, Amsterdam, 1985, 163-184.

Klaiber, A., and M. Gokhale [1992]. Parallel evaluation of attribute grammars. IEEE Transactions on Parallel and Distributed Systems 3(2), 1992, 206-220.

Klein, P.N., and J.H. Reif [1988]. Parallel time $O(\log n)$ acceptance of deterministic CFLs on an exclusive-write P-RAM. SLAM Journal on Computing 17, 1988, 463-485.

Klein, E.F., and K. Koskimies [1989]. The parallelization of one-pass compilers. Arbeitspapiere der GMD 416, Gesellschaft fur Mathematik und Datenverarbeitung mbH, Karlsruhe, Germany, November 1989

Klein, E.F. [1990]. Attribute evaluation in parallel. Proceedings of the Workshop on Parallel Compilation, Kingston, Ontario, Canada, May 1990.

Klein, E.F., and K. Koskimies [1990]. Parallel one-pass compilation. Proceedings of the WAGA '90 International Workshop on Attribute Grammars and their Applications, Paris, France, September 1990, Lecture Notes in Computer Science 461, Springer Verlag, Berlin, Germany, 76-90.

Klein, E.F. [1991]. Ein Modell zur Generierung paralleler Attributauswerter. Dissertation, Fakultäı für Informatik, Universităt Karlsruhe, Karlsruhe, Germany, 1991.

Klein, E.F., and K. Koskdmies [1991]. A method to pipeline parsing with parallel semantic analysis. Arbeitspapiere der GMD 506, Gesellschaft for Mathematik und Datenverarbeitung mbH, Karlsruhe, Germany, January 1991.

Klein, E.F. [1992]. Parallel ordered attribute grammars. Proceedings of the IEEE Computer Society 1992 International Conference on Computer Languages, Oakland, CA, USA, April 20-23, 1992, 106-116. 
Kosaraju, S.R. [1975]. Speed of recognition of context-free languages by array automata. SLAM Journal on Computing 4, 1975, 331-340.

Krobn, H.E. [1975]. A parallel approach to code generation for Fortran-like compilers. Proceedings of the Conference on Programming Languages and Compilers for Parallel and Vector Machines. SIGPLAN Notices 10(3), 1975, 146-152.

Kuiper, M.F. [1988]. Increasing parallelism in attribute grammar evaluators: transformations and lazy evaluation. Proceedings Computer Science in the Netherlands, CSN 88 SION, part I, 1988, 109-121.

Kuiper, M.F. [1989]. Parallel attribute evaluation. Ph.D. Thesis, University of Utrecht, 1989. Kuiper, M.F., and A. Dijkstra [1989]. Attribute evaluation on a network of transputers. In Developing Transputer Applications, J. Wexler (ed.), Amsterdam, 1989, 142-149.

Kuiper, M.F., and S.D. Swierstra [1990]. Parallel attribute evaluation: structure of evaluators and detection of parallelism. Proceedings of the WAGA ' 90 International Workshop on Attribute Grammars and their Applications, Paris, France, September 1990, Lecture Notes in Computer Science 461, Springer Verlag, Berlin, Germany, 61-75.

Ladner, R.E., and M.J. Fischer [1980]. Parallel prefix computation. Journal of the ACM 27(4), October 1980, 831-838.

Lampe, J. [1988]. Automatische Generierung von Fachsprachprocessoren mit Ereignisgesteuerter Allributbewerlung. Proceedings INFO'88 Berlin, February 1988, 134-135.

Lompe, J. I1990al. MATE - A melasystem with concurrent attribute evaluation. Proceedings Hierkishop inn Cimpuler Cimputers, Schwerin, Germany, Octuber 22-24, 1990, 220-230.

Immpe, J. [19906]. Zur purallelen Verarteilung furmal detinierter Sprachen. Habilitationsthesis, Technix he Universital Drescen. Dresckn, Germany, December 1990, in German.

Lang, B. | 1971]. Parallet non-deterministic txottom-up parsing. Proceedings of the International Symposium on Exitensible Languages. Grenoble, 1971, S/GPLAN Notices 6(12), 1971.

Langlois, L.C. [1988]. Paraltel parsing of context-free languages on an array of processors. Ph.D. Thesis, CST 54-88, Department of Computer Science, University of Edinburgh, Scotland, October 1988.

Langlois, L.C. [1990]. Systolic parsing of context-free languages. International Journal on Parallel Programming 19(4), 1990, 333-335.

Larmore, L.L., and W. Rytter [1992]. Efficient sublinear time parallel algorithms for dynamic programming and context-free recognition. Proceedings of the 9th Annual Symposium on Theoretical Aspects of Computer Science, STACS'92.

Lee, C.-I., and S.-H. Kim [1990]. Parallel syntactic processing with a chart. Seoul International Conference on Natural Language Processing, Language Research Institute, Seoul National University, Seoul, South Korea, 1990, 38-42.

Lee, Y.-F., T.J. Marlowe, and B.G. Ryder [1990]. Paraliel data-flow analysis algorithms. Proceedings, Workshop on Parallel Compilation, Kingston, Ontario, Canada, May 6-8, 1990

Lee, Y-F., B.G. Ryder, and T.J. Marlowe [1991]. Experiences with a parallel algorithm for data-flow analysis. Journal of Supercomputing 5(2/3), 1991, 163-188.

Lewke, K.-D. [1990]. Pila: Lexical and syntactical analysis on a pipelined processor. Proceedings of the Workshop on Parallel Compilation, Kingston, Ontario, Canada, May 6-8, 1990.

Ligett, D., G. McCluskey, and W.M. McKeeman [1982]. Parallel LR parsing. Technical Report TR-82-03. Wang Institute of Graduate Studies, School of Information Technology, 1982.
Lincoln, N. [1970]. Parallel programming techniques for compilers. SIGPLAN Notices 5(10), $1970,18-31$

Lipkie, D.E. [1979]. A compiler design for multiple independent processor computers. Ph.D. Thesis Department of Computer Science, University of Washington, Seattle, WA, 1979.

Loka, R.R. [1984]. A note on parallel parsing. SIGPLAN Notices 19(1), 1984, 57-59.

Lozinskii, E.L., and S. Nirenburg [1986]. Parsing in parallel. Computer Languages 11, 1986, 39-51.

Marmol, B. [1990]. Evaluateurs d'attributes paralteles sur multi-processeurs à mémoire partagée. Rapport de DEA, University of Ortécuans, September 1990.

Matsumoto, Y., H. Tanaka, H. Hirakawa, H. Miyoshi, and H. Yasukawa [1983]. BUP: A bottom-up parser embedded in Prolog. New Generation Computing 1, 1983, 145-158.

Matsumoto, Y. [1987]. A parallel parsing system for natural language analysis. New Generation Computing 5, 1987, 63-78.

Matsumoto, Y., and R. Sugimura [1987]. A parsing system based on logic programming. Proceedings of $I J C A I-87,1987,671-674$.

Mattheyses, R., and C.M. Fiduccia [1982]. Parsing Dyck languages on parallel machines. Proceedings of the 20th Allerton Conference on Communication, Control and Computing, 1982, 272-280.

McDonald, I.D., and A.L. Wendelborn [1989]. Parallel lexical analysis. Proceedings of the Workshop on Parallel Compilation, Kingston, Ontario, Canada, May 1990.

Messerer, M. [1982]. Ein never Ansatz zur Parallelisierung von Compilern. Ph.D. Thesis, Arbeitsberichte des Institutes für mathematische Maschinen und Datenverarbeitung (Informatik) Band 15, Nr. 8, Universitä0 Erlangen, 1982.

Mickunas, M.D., and R.M. Schell [1978]. Parallel compilation in a multi-processor environment. Proceedings Annual Conference ACM, 1978, 241-246.

Miller, J.A., and R.J. LeBlanc [1982]. Distributed compilation: a case study. Proceedings of the Third International Conference on Distributed Computing Systems, 1982, 548-553.

Mukhopadhyay, A. [1979]. Hardware algorithms for nonnumerical computation. IEEE Transac tions on Computers C-28(6), 1979, 384-394.

Nijholt, A. [1989]. Parallel parsing strategies in natural language processing. Proceedings of the International Workshop on Parsing Technologies, Carnegie Mellon University, Pittsburgh, August $1989,240-253$.

Nijholt, A. [1990]. Meta-parsing in neural networks. Tenth European Meeting on Cybernetics and Systems Research, World Scientific Publishing Corp., Austrian Society for Cybernetic Studies, Vienna, 1990.

Nijholt, A. [1993]. Parallel approaches to context-free language parsing. In: Parallel Models of Natural Language Computation, U. Hahn and G. Adriaens (eds.), Ablex Publishing Corporation, Norwood, New Jersey, summer 1993.

Numazaki, H., and H. Tanaka [1990]. A new parallel algorithm for generalized LR parsing. Proceedings of the 13th International Conference on Computational Linguistics (COLING'90), Helsinki, Vol. 2, 304-310.

Oude Luttighuis, P.H.W.M. [1989]. Paraltel parsing of regular right-part grammars. Memoranda Informatica INF 89-63, University of Twente, Enschede, The Netherlands, December 1989.

Oude Luttighuis, P.H.W.M. [1990a]. Parallel parsing of regular right-part grammars. Proceedings of the Workshop on Parallel Compilation, Kingston, Ontario, Canada, May 1990. 
Oude Luttighuis, P.H.W.M. [1990b]. Private communication with David Skillicorn and David Barnard, 1990.

Oude Luttighuis, P.H.W.M. [1991]. Optimal parallel parsing of almost all LL( $k$ ) grammars. Memoranda Informatica INF 91-78, Department of Computer Science, University of Twente Enschede, The Netherlands, 1991.

Oude Luttighuis, P.H.W.M. [1993]. Parallel algorithms for parsing and attribute evaluation. Ph.D. Thesis, University of Twente, Enschede, The Netherlands, June 1993.

Oude Luttighuis, F.H.W.M., and N. Sikkel [1992]. Attribute evaluation during generalized parsing. Memoranda Informatica INF 92-85, University of Twente, Enschede, The Netherlands, December 1992.

Palis, M., and S. Shende [1988]. Upper bounds on recognition of a hierarchy of non-context-free languages. Techn. Report MS-CIS-88-56. Department of Comptiting and Information Science, School of Engineering and Applied Science, Philadelphia, PA, USA, 1988.

Palis, M., and S. Shende [1989]. Sublinear parallel time recognition of tree adjoining languages. Proceedings of the 1989 International Conference on Parallel Processing, August 8-12, 1989, University Park, PA, USA, Vol. III (Algorithms and Applications), F. Riggs and M. Kogge (eds.) August 1989.

Palis, M., S. Shende and D. Weir [1990]. An optimal linear-time parallel parser for tree-adjoining languages. SLAM Journal on Computing 19(1), 1-31.

Partsch, H. [1983]. A transformational approach to parsing and recognition. Technical Report, Institut für Informatik der TU Munchen, 1983.

Partsch, H. [1984a]. Structuring transformational developments: a case study based on Earley's recognizer. Science of Computer Programming 4, 1984, 17-44.

Partsch, H. [1984b]. Transformational derivation of parsing algorithms executable on paralle architectures. In: Programmiersprachen und Programmentwicklung, 8. Fachtagung, Zürich, März 1984, Informatik Fachberichte 77, U. Ammann (ed.), Springer Verlag, Berlin, Germany, 1984, 41-57.

Pippenger, N. [1979]. On simultaneous resource bounds. Proceedings of the 20th IEEE Symposium on Theory of Computing, 1979, 307-311.

Pitsch, G., and E. Schomer [1991]. Optimal parallel recognition of bracket languages on hypercubes. Proceedings of the 8th Annual Symposium on Theoretical Aspects of Computer Science, STACS'91, Hamburg, Germany, February 1991, Lecture Notes in Computer Science 480, Springer Verlag, Berlin, Germany, 434-444.

Prasad, S.K., and N. Deo [1991]. Two EREW algorithms for parentheses matching. Submitted to: Fifth International Parallel Processing Symposium, 1991.

Pronina, V.A., and E.A. Trakhtengerts [1974]. Parallel syntax analysis. Automation and Remote Conirol 35(7), Part 1, 1974, 1118-1122.

Pronina, V.A., and A.A. Chudin [1975]. Syntax analysis implementation in an associative paralle! processor. Automation and Remote Control 36(8), 1975, 1303-1308.

Reif, J.H. [1982]. Parallel time $O(\log n)$ acceptance of deterministic CFLs. Proceedings of the 23rd IEEE Conference on Fundamentals of Computer Science, 1982, 290-296.

Ruzzo, W.L. [1979]. Tree-size bounded alternation. Journal of Computer and System Sciences $21,1980,218-235$.

Ruxeo, W.L. [1981]. On uniform circuit complexity. Journal of Computer and System Sciences 22. $1981,365-383$
Rytter, W. [1985a]. On the recognition of context-free languages. Proceedings of the Fifth Symposium on Computation Theory. A. Skowron (ed.), Lecture Notes in Computer Science 208 , Springer Verlag, Berlin, Germany, 1985, 318-325.

Rytter, W. [1985b]. The complexity of two-way pushdown automata and recursive programs. In: Combinatorial algorithms on words, A. Apostolico and Z. Galil (eds.), Springer Verlag, Germany, 1985, 341-356.

Rytter, W. [1986a]. An application of Mehlhorn's algorithm for bracket languages to $\log n$ space recognition of input-driven languages. Information Processing Letters 23, 1986, 81(mi84.

Rytter, W. [1987a]. On the complexity of parallel parsing of general context-free languages. Theoretical Computer Science 47, 198\%, 315-321.

Rytter, W. [1987b]. Parallel time $O(\log n)$ recognition of unambiguous CFLs. Information and Computation, 73, 1987, 75-86.

Rytter, W., and R. Giancarlo [1987]. Optimal parallel parsing of bracket languages. Theoretical Computer Science 53, 1987, 295-306.

Sarkar, D., and N. Deo [1985]. Estimating the speed-up in parsing. Report CS-85-135, Computer Science Department, Washington State University, WA, USA, 1985.

Sarkar, D., and N. Deo [1987]. An optimal parallel parsing algorithm for a class of blockstructured languages. Proceedings of the 1987 International Conference on Parallel Processing, August 1987, 585-588.

Sarkar, D., and N. Deo [1988]. Parallel algorithms for parentheses matching and generation of random balanced sequences of parentheses. Pruceedings of the 1st International Conference on Supercomputing, Athens, Greece, June 1987, E.N. Houstis, T.S. Papatheodorou, and C.D. Polychronopoulos (eds.), Lecture Notes in Computer Science 297, Springer Verlag, Berlin, Germany, 1988, 970-984.

Schell Jr., R.M. [1979]. Methods for constructing parallel compilers ior use in a multi-processor environment. Ph.D. Thesis, Department of Computer Science, Report No. 958, University of Illinois at Urbana-Champaign, Urbana, IL, USA, February 1979.

Schmeiser, J.P. [1992]. Polylogarithmic parallel parsing, Ph.D. Thesis, Queen's University, Kingston, Ontario, Canada, September 1992.

Seo, K. M. Hirayama, and A. Fusaoka [1983]. Design and evaluation of parsing chip. VLS '83, F. Anceau and E.J. Aas (eds.), Elsevier Science Publishers B.V. (North-Holland), Amsterdam, $1983,317-326$.

Seshadri, V. [1988a]. Concurrent semantic analysis. CSRI 216, Computer Systems Research Institute, University of Toronto, Toronto, Ontario, Canada, September 1988

Seshadri, V., I.S. Small, and D.B. Wortman [1988b]. Concurrent compilation. Proceedings of the IFIP WG103 Working Conference on Distributed Processing, M.H. Barton, E.L. Dagless, and G.L. Reijns (eds.), North Holland, 1988, 627-641.

Seshadri, V., D.B. Wortman, M.D. Junkin, S. Weber, C.P. Yu, and I.S. Small [1988c]. Semantic analysis in a concurrent compiler. Proceedings of the SIGPLAN ' 88 Conference on Programming Language Design and Implementation, SIGPLAN Notices 23(7), 1988, 233-240.

Seshadri, V., and D.B. Wortman [1991]. An investigation into concurrent semantic analysis Software-Practice and Experience 21(12), 1991, 1323-1348.

Sijstermans, F.W. [1986]. Parallel parsing of context-free languages. Doc. No. 202, Esprit Project 415, Subproject A: Object-oriented language approach, Philips Research Laboratories, Eindhoven, 1986 
Sikkel, K. [1990]. Cross-fertilization of Earley and Tomita. Memoranda Informatica INF 90-69, Department of Computer Science, University of Twente, Enschede, The Netherlands, 1990. Sikkel, K. [1991]. Parallel on-line parsing in constant time. Memoranda Informatica INF 91-64, Department of Computer Science, University of Twente, Enschede, The Netherlands.

Sikkel, K., and A. Nibolt [1991]. Connectionist parsing of context-free grammars. 2nd International Workshop on Parsing Technologies (IWPT'91), Cancun, Mexico, 117-126.

Sikkel, K., and M. Lankhorst [1992]. A parallel bottom-up Tomita parser. In: G. Görz (Hrsg.), 1. Konferenz Verarbeitung natürlicher Sprache (KONVENS'92), Nürnberg, Germany. Informatik Aktuell, Springer-Verlag, Berlin, Germany, 1992, 238-247.

Skillicorn, D.B., and D.T. Barnard [1989]. Parallel parsing on the Connection Machine. Information Processing Letters 31, 1989 11-117.

Skillicorn, D.B., and D.T. Barnard [1990a]. Parallel compilation: A status report. External Technical Report ISSN-0836-0227-90-267, Department of Computing and Information Science, Queens's University, Kingston, Ontario, Canada, March 27, 1990.

Skillicorn, D.B., and D.T. Barnard [1990b]. A survey of parallel compilation. Department of Computing and Information Science, Queens's University, Kingston, Canada, September 12, 1990. Sommerhalder, R., and S. van Westrhenen [1983]. Parallel language recognition in constant time by cellular automata. Acta Informatica 19, 1983 397-407.

Sridharan, N.S. [1987]. Semi-applicative programming: examples of context-free recognizers. Chapter 8 in: Distributed Artificial Intelligence, M.N. Huhns (ed.), Research Notes in Artificial Intelligence, Morgan Kaufmann Publishers, Inc., Los Altos, CA, USA, 1987, 203-245.

Srikent, Y.N., and P. Shankar [1907a]. Parullel parsing of programming languages. Information Sitences $43,1467,55-k 3$.

Srikant, Y.N., and P. Shankar (1987b). A new parallel algorithm for parsing arithmetic infix expressions. Purallel Computing 4, 1987, 291-304.

Srikant, Y.N. [1990]. Parallel parsing of arithmetic expressions. IEEE Transactions on Computers 39(1), January 1990, 310-312.

Steele, G.L., and W.D. Hillis [1986]. Connection machine Lisp: Fine-grained parallel symbolic processing. Proceedings of the 1986 ACM Conference on Lisp and Functional Programming, Cambridge, MA, USA, 1986, 279-297.

Tan, H.D.A. [1983]. VLSI-algoritmen voor herkenning van context-vrije talen in lineaire tijd. Rapport IN 24/83, Stichting Mathematisch Centrum, Amsterdam, The Netherlands, June 1983, in Dutch.

Tanaka, H., and H. Numazaki [1989]. Parallel generalized LR parsing based on logic programming. Proceedings International Workshop on Parsing Technologies (IWPT'89), Carnegie Mellon University, Pittsburgh, PA, USA, 329-338.

Thompson, H.S. [1989]. Chart parsing for loosely coupled parallel systems. Proceedings of the International Workshop on Parsing Technologies, Carnegie Mellon University, Pittsburgh, PA, USA, August 1989, 320-328.

Thompson, H.S., M. Dixon, and J. Lamping [1991]. Compose-reduce parsing. 29th Annual Meeting of the ACL, Berkeley, CA, USA, 87-97.

Tomita, M. [1985]. Efficient Parsing for Natural Language. Kluwer Academic Publishers, 1985 Tsang, W.W., T.W. Lam, and F.Y.L. Chin [1989]. An optimal EREW P-RAM parallel algorithm for parentheses matching. Proceedings of the 1989 International Conference on Paralle Processing, University Park, PA, USA, Vol. Ill (Algorithms and Applications), August 1989
185-192.

Tseytlin, G.E., and E.L. Yushchenko [1977]. Several aspects of theory of parametric models of languages and parallel syntactic analysis. In: Methods of Algorithmic Language Implementation, A. Ershov and C.H.A. Koster (eds.), Lecture Notes in Computer Science 47, Springer Verlag, Berlin, Germany, 1977, 231-245.

Vandevoorde, M.T. [1988a]. Parallel compilation on a tightiy coupled multi-processor. SRC Reports 26, Digital Systems Research Center, March 1988.

Vandevoorde, M.T., and E. Roberts [1988b]. WorkCrews: An abstraction for controlling parallelism. International Journal of Parallel Programming 17(4), 1988, 347-366.

Vreught, J.P.M. de, and H.J. Homig [1989]. A tabular bottom-up recognizer. Delft University of Technology, Faculty of Technical Mathematics and Informatics. Intern Rapport TWI 89-78, 1989. Vreught, J.P.M. de, and H.J. Honig [1990a]. A fast parallel recognizer. Delft University of Technology, Faculty of Technical Mathematics and Informatics. Intern Rapport TWI 90-16.

Vreught, J.P.M. de, and H.J. Honig [1990b]. General context-free parsing. Delft University of Technology, Faculty of Technical Mathematics and Informatics. Intern Rapport TWI 90-31.

Vreught, J.P.M. de [1991]. An annotated bibliography on parallel parsing. Report 91-64, Delf University of Technology, Delft, The Netherlands, 1991.

Vreught, J.P.M. de, and H.J. Honig [1991]. Slow and fast parallel recugnition. Proceedings of the Second International Workshop on Pursing Technologies (IWPT'91), Cancun, Mexico, 1991 127-135.

Viswanathan, N., and Y.N. Srikant [1991]. Parallel incremental LR parsing. Proceedings of the 1991 International Conference on Parallel Processing, Austin, TX. USA, vol. III (Algorithms and Applications), August 1991, 328-329.

Wortman, D.B. [1990]. A concurrent Modula-2+ compiler. Proceedings Workshop on Paralle Compilation, Kingston, Ontario, Canada, May 6-8, 1990.

Wortman, D.B., and M.D. Junkin [1992]. A concurrent compiler for Modula-2+. Proceedings of the SIGPLAN '92 Conference on Programming Language Design and Implementation, SIGPLAN Notices 27(7), 1992, 68-81.

Yonezawa, A., and I. Ohsawa [1989]. Object-oriented parallel parsing for context-free grammars. In: ABCL: an Object-Oriented Concurrent System, A. Yonezawa (ed.), The MIT Press, 1989. Zaring, A.K. [1990]. Parallel evaluation in attribute grammar-based systems. Ph.D. thesis, Department of Computer Science, Cornell University, Ithaca, NY, USA, 1990.

Zobel, A. [1990]. Parallelized compiler optimization. Proceedings Workshop on Parallel Compilation, Kingston, Ontario, Canada, May 6-8, 1990.

Zosel, M. [1973]. A parallel approach to compilation. Proceedings of the ACM Symposium on Principles of Programming Languages, 1973, 59-70. 